\title{
Interplay of structural and magnetic nanoscale phase separation in layered cobaltites
}

\author{
M. Allieta, ${ }^{1,{ }^{*}}$ M. Scavini,,${ }^{1,2}$ A. Naldoni, ${ }^{3}$ M. Coduri ${ }^{4}$ S. Cappelli, ${ }^{1}$ C. Oliva,${ }^{1,2}$ S. Santangelo, ${ }^{5}$ C. Triolo, ${ }^{6}$ S. Patané, ${ }^{6}$ \\ A. Lascialfari, ${ }^{7,8}$ and V. Scagnoli ${ }^{9,10}$ \\ ${ }^{1}$ Università Degli Studi di Milano, Dipartimento di Chimica, Via C. Golgi 19, I-20133 Milano, Italy \\ ${ }^{2}$ CNR-ISTM and INSTM, Unit Milan, Via C. Golgi 19, I-20133 Milan, Italy \\ ${ }^{3}$ CNR-Istituto di Scienze e Tecnologie Molecolari, Via C. Golgi 19, I-20133 Milan, Italy \\ ${ }^{4}$ Istituto per l'Energia e le Interfasi, CNR-IENI, C. so Promessi Sposi 29, 23900 Lecco, Italy \\ ${ }^{5}$ Dipartimento di Ingegneria Civile, dell'Energia, dell'Ambiente e dei Materiali (DICEAM), Università Mediterranea, \\ 89122 Reggio Calabria, Italy \\ ${ }^{6}$ Dipartimento di Fisica e di Scienze della Terra (DFST), Università di Messina, 98166 Messina, Italy \\ ${ }^{7}$ Dipartimento di Fisica and INSTM Unit, Università Degli Studi di Milano, 20133 Milano, Italy \\ ${ }^{8}$ Dipartimento di Fisica, Università Degli Studi di Pavia, 27100 Pavia, Italy \\ ${ }^{9}$ Laboratory for Mesoscopic Systems, Department of Materials ETH Zurich, 8093 Zurich, Switzerland \\ ${ }^{10}$ Laboratory for Micro- and Nanotechnology, Paul Scherrer Institut, CH-5232 Villigen PSI, Switzerland
}

(Received 5 April 2015; published 11 August 2015)

\begin{abstract}
We report on the structural, electronic, and magnetic phases of a previously unexplored region in the phase diagram of $\mathrm{GdBaCo}_{2} \mathrm{O}_{5+\delta}(\delta=0.57$ and 0.63$)$. Despite a homogenous average structure displayed by both the samples, the orthorhombic highly oxygenated $\mathrm{GdBaCo}_{2} \mathrm{O}_{5.63}$ shows clear signatures of structural nanoscale phase separation. By combining a pair distribution function with photoluminescence and electron spin resonance techniques, we found that the nanoscale phase separation is induced by an inhomogeneous distribution of ferromagnetic $\mathrm{Co}^{3+}-\mathrm{Co}^{4+}$ clusters embedded in an antiferromagnetic $\mathrm{Co}^{3+}$-rich matrix. In addition, we uncovered a phase evolution involving the collapse of the orthorhombic strain below room temperature. The origin of this noncanonical transition seems to be associated with the interplay of the observed nanoscale phase separation and a new magnetic phase transition occurring below $T \sim 180 \mathrm{~K}$.
\end{abstract}

DOI: 10.1103/PhysRevB.92.054202

\section{INTRODUCTION}

The structure of strongly correlated electron oxides varies dramatically with spatial scales leading to unusual phase transitions triggered by temperature [1-4]. The long-range spatial and/or magnetic correlations have quite often little relevance in determining the material physical properties. Local atomic fluctuations promote broken symmetry states $[3,4]$, nanoscale phase separations [1,2], and reentrant phase transitions [4] associated with emergent phenomena, such as colossal magnetoresistance (MR) and high-temperature superconductivity $[1,2]$. Therefore the interplay between the structure and such inhomogeneous phases plays a pivotal role in the understanding the physics of the strongly correlated electron oxides at the microscopic level [3-9].

Layered cobaltite $\mathrm{GdBaCo}_{2} \mathrm{O}_{5+\delta}$ (GBCO) is an ideal system to investigate the role of nanoscale phase separation due to its intrinsic inhomogeneous structure [4-6,10-12]. Giant MR effects [11], magnetic ordering [12], charge ordering [4], and metal-insulator transition (MIT) [6-12] coexist within the same material without requiring extrinsic chemical doping. In the optimal oxygen $\delta$ range $0.45 \leqslant \delta \leqslant 0.55$, the structure of $\mathrm{GBCO}$ consists of layers $\left[\mathrm{CoO}_{2}\right][\mathrm{BaO}]\left[\mathrm{CoO}_{2}\right]\left[\mathrm{GdO}_{0.5}\right]$ stacking along the $c$ axis of the Pmmm space group with a $a \times 2 a \times 2 a$ cell metric, where $a$ is the primitive cubic perovskite lattice parameter [6]. In the $\mathrm{GdO}_{0.5}$ plane the oxygen site $1 c(0,0,1 / 2)$ is almost empty whereas the oxygen site $1 g(0,1 / 2,1 / 2)$ is fully occupied, giving rise to two types of coordination environments for cobalt ions, i.e.,

*mattia.allieta@gmail.com
PACS number(s): 64.60.My, 61.05.cp, 64.70.K-, 76.30.-v

pyramidal $\left(\mathrm{CoO}_{5}\right)$ and octahedral $\left(\mathrm{CoO}_{6}\right)$ [6]. Within this Pmmm phase GBCO shows several transitions, namely, a MIT below $T_{\mathrm{MIT}} \sim 350 \mathrm{~K}$ in the paramagnetic $(\mathrm{PM})$ phase, a PM-to-ferromagnetic (FM) transition at $T_{\mathrm{C}} \sim 279 \mathrm{~K}[11,12]$, and a FM-to-antiferromagnetic (AFM1) transition at $T_{\mathrm{N} 1} \sim$ $255 \mathrm{~K}[11,12]$ which is associated to the onset of giant MR. Exceeding $\delta=0.55$, the system develops a magnetic transition series but the structure is characterized by the coexistence of regions with different $\delta$ levels, inducing a long-range phase separation [12]. At $\delta \geqslant 0.70$, the system recovers its magnetic homogeneity featuring a new series of magnetic transitions, namely, PM - FM2 and FM2 - AMF3 at $T \sim 180 \mathrm{~K}$ and $T \sim 100 \mathrm{~K}$, respectively [12].

There have been several efforts to study the interplay of structure and physical properties in the optimally doped layered cobaltites [6,8-14], but direct measurements to support the phase inhomogeneities in the $0.55 \leqslant \delta<0.70$ range have yet to be realized. Previous neutron powder diffraction (NPD) studies on $\mathrm{LnBaCo}_{2} \mathrm{O}_{5+\delta}(\mathrm{Ln}=$ lanthanide $)$ have revealed the spin configurations [15] and even the coexistence of different magnetic phases [16] as a function of temperature. In Gd-based compounds the high-absorption cross section of $\mathrm{Gd}$ in its natural abundance isotopic composition has so far precluded neutron diffraction studies on that system.

In this paper, we report on the interplay among the structural and magnetic properties of $\delta=0.54,0.63$ samples by using a combination of synchrotron powder diffraction, photoluminescence (PL), magnetic susceptibility, and electron spin resonance (ESR) techniques. In particular, ESR, being sensitive to strong Gd-Gd dipolar interactions, is a very powerful tool to study the magnetism in natural Gd-based compounds. We found for $\delta=0.63$ material a structural 
transition which involves a continuous decrease of its orthorhombic strain on cooling. This unusual behavior is driven by a nanoscale magnetic phase separation resulting in the formation of FM clusters within the AFM1 matrix. ESR measurements are consistent with a previously unreported AFM1-AFM2 transition occurring in both $\delta=0.54$ and $\delta=$ 0.63 samples at low temperature.

\section{EXPERIMENTAL TECHNIQUES AND METHODS}

GBCO samples were synthesized by conventional solidstate reaction techniques [6]. The desired oxygen content was adjusted according to annealing conditions and thermal treatments reported by Taskin et al. [12], and $\delta$ values were determined by the procedure used in our previous study [6].

High-resolution synchrotron powder diffraction experiments were performed at the ID31 beamline of the European Synchrotron Radiation Facility by selecting $\lambda=0.39620(5) \AA$. Patterns in the $0^{\circ} \leqslant 2 \vartheta \leqslant 50^{\circ}$ range data were collected for 1 hour counting time between 5 and 298 $\mathrm{K}$. Additionally, for the $\delta=0.63$ sample more patterns were collected in the $70 \mathrm{~K} \leqslant T \leqslant 250 \mathrm{~K}$ range every $3 \mathrm{~K}$. The sample was cooled to $80 \mathrm{~K}$ by using a $\mathrm{N}_{2}$ gas blower (Oxford Cryosystems), whereas a liquid-helium-cooled cryostat was employed to achieve the lowest temperature. In particular, at $T=298 \mathrm{~K}$ several scans were summed up for $7 \mathrm{~h}$ counting time $\left(Q_{\max } \approx 27 \AA^{-1}\right)$ to achieve statistical significance for pair distribution function (PDF) analysis.

Rietveld refinements were performed using the GSAS software [17]. PDF analysis of the powder diffraction data collected at $T=298 \mathrm{~K}$ was carried out using the formalism of $G(r)$ functions as implemented in PDFGETX2 software [18]. PDF were modeled using PDFGUI [19] by determining the degree of accuracy of the refinement by the agreement factor $R_{w}$ as defined in Ref. [19].

$\mathrm{PL}$ analysis in the $50 \mathrm{~K} \leqslant T \leqslant 300 \mathrm{~K}$ range was performed on pellets obtained by pressing the powder materials. No other pretreatment was necessary. The experimental setup consisted of a cold finger helium-based cryostat (Leybold Cryotip) equipped with a two-stage cold head. The sample holder, located within an aluminum chamber, was designed ad hoc to easily host the tablet assuring a homogeneous temperature distribution. A Cryogenic linear temperature sensor glued to the sample holder and connected to a multimeter (3457A Hewlett Packard) monitored the temperature inside the cryostat. The incident light on the sample surface was via a sapphire window and was focalized by a $10 \times$ optical objective installed on the revolver of a metallographic microscope (NIKON). A Nd:YAG laser installed in the lamp port of the microscope provided $532-\mathrm{nm}(2.33 \mathrm{eV})$ excitation. The same objective used to excite the sample collected the PL emission in reflection mode. A set of suitable filters cut off the laser light and allowed the signal to reach the microscope camera port, where a lens coupled the light to a multimode optical fiber connected to a spectrometer (DKSP480 CVI). A thermoelectric cooled CCD camera (Andor iDus) was used as a detector.

Magnetic characterization was made by performing ESR measurements at a Bruker ELEXSYS spectrometer equipped with an ER4102ST standard rectangular cavity at $X$ band $(9.4 \mathrm{GHz})$ frequency every $5 \mathrm{~K}$ in the temperature range
$120 \mathrm{~K} \leqslant T \leqslant 300 \mathrm{~K}$. The derivative $d P / d H$ of power $P$ absorbed was recorded as a function of the static magnetic field $H$. Magnetic susceptibility $\left(\chi_{\mathrm{m}}\right)$ measurements were performed with a MPMS-XL7 Quantum Design superconducting quantum interference device (SQUID) magnetometer in the temperature range $2-300 \mathrm{~K}$ at 20 Oe constant magnetic field.

\section{RESULTS AND DISCUSSION}

At room temperature both samples are well described by the orthorhombic structural model Pmmm [6]. However, as exemplified in Fig. 1(a), for the $\delta=0.63$ sample it is clearly noticeable that upon cooling the (040) and (200) peaks merge into a singlet at low temperature. This effect is more evident at higher $q$ values where, e.g., orthorhombic $(h k l)$ and $(k h l)$ pairs of diffraction peaks are included within well-shaped single $(h k l)$ peaks in the $T=150 \mathrm{~K}$ pattern [Fig. 1(b)]. Below $T \sim 150 \mathrm{~K}$ the merged (040), (200) peaks split again and keep an asymmetric shape down to $5 \mathrm{~K}$ [Fig. 1(a)]. The structural transition is therefore revealed by a continuous modification of $d$ spacing related to the unit cell axes. This can be directly quantified by the orthorhombic strain defined as $\eta=$ $2(a-b / 2) /(a+b / 2)$, which results to be zero for a tetragonal unit cell. The strain values for $\delta=0.63$ and $\delta=0.54$ are reported in Fig. 1(c). Below room temperature, $\eta$ in $\delta=0.54$ shows a rather weak monotonic temperature dependence down to $5 \mathrm{~K}$, while for $\delta=0.63$ the strain dramatically decreases, reaching a minimum $(\eta \sim 0.16 \%)$ at $T=150 \mathrm{~K}$ with a less pronounced variation below this temperature [Fig. 1(c)]. Within the high resolution achieved in our synchrotron
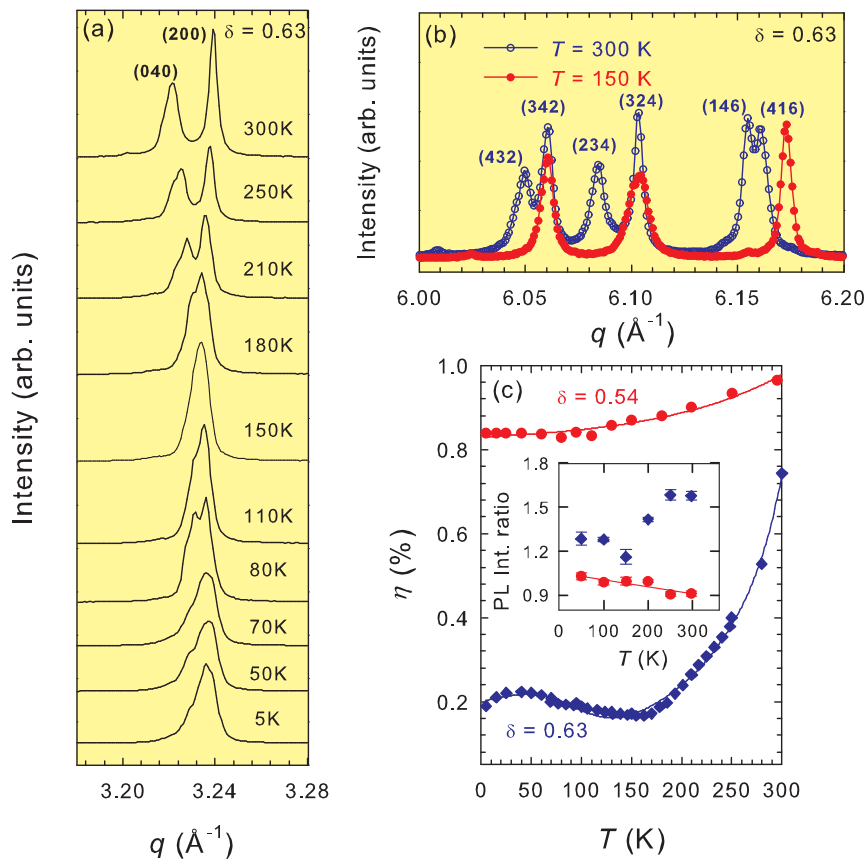

FIG. 1. (Color online) (a) Temperature evolution of (040), (200) diffraction peaks for the $\delta=0.63$ sample. (b) Magnified view of patterns collected at $T=300 \mathrm{~K}$ (empty circles) and $T=150 \mathrm{~K}$ (full circles). (c) $\eta(\%)$ as a function of temperature. Inset to (c) reports the temperature dependence of photoluminescence intensity ratio. Full circles and diamonds in panels are data related to $\delta=0.54, \delta=0.63$ samples. 

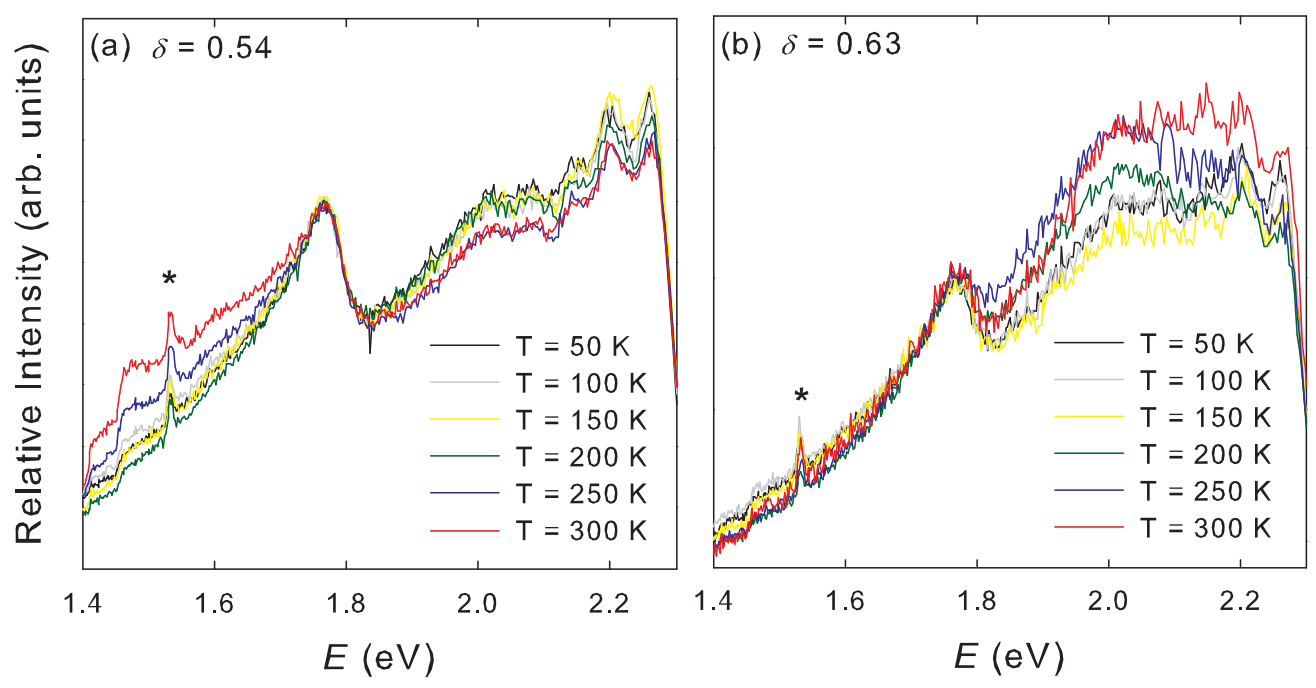

FIG. 2. (Color online) Temperature dependence of photoluminescence spectra for samples (a) $\delta=0.54$ and (b) $\delta=0.63$. The spectra are normalized to the maximum intensity of the peak at $1.77 \mathrm{eV}$ for an easier comparison. Star marks the spurious peak due to the Nd:YAG laser pump at $1.53 \mathrm{eV}$.

powder diffraction experiments $\left(\Delta d / d=10^{-3}-10^{-4} \AA\right)$, the evolution of $\eta$ provides evidence of a true collapse of the orthorhombic strain upon cooling of the high-temperature orthorhombic Pmmm structure. In the canonical view of continuous phase transition, the structural phase keeps a group-subgroup relationship, and for a temperature-driven transition the symmetry of the low-temperature phase is lower than the symmetry of the high-temperature phase. Although we did not find a clear symmetry breaking of the Pmmm model upon cooling, the continuous structural evolution reported here seems to suggest an opposite trend.

We first studied the relationship between the phase transition and the electronic structure by PL technique. Figures 2(a) and 2(b) show PL spectra of the investigated samples for various temperatures in the $50-300 \mathrm{~K}$ range. Two bands peaking at 1.77 and $2.02 \mathrm{eV}$ dominate all the spectra. In the case of sample $\delta=0.54$ [Fig. 2(a)], the relative intensities do not significantly vary with temperature. Conversely, in the case of sample $\delta=0.63$ [Fig. 2(b)], the relative intensity of the band at higher energy is temperature dependent.

To the best of our knowledge, the PL spectra of layered perovskite $\mathrm{GdBaCo}_{2} \mathrm{O}_{5+\delta}$ have never been reported in literature. According to Pashkevich et al. [20], Co oxides, present at intercrystallite boundaries and on the surface of the samples, are responsible for the strong luminescence that hinders the detection of Raman signals in polycrystalline $\mathrm{GdBaCo}_{2} \mathrm{O}_{5+\delta}$. Indeed, a broad green emission band is associated to the presence of oxygen vacancy defects in the near-surface region of transition-metal oxides and transition-metal-doped materials [20-25]. Witek et al. [26] reported the detection of a PL band around $1.78 \mathrm{eV}$ in Gd-doped high-quality bulk GaN and identified the ${ }^{6} G_{7 / 2} \rightarrow{ }^{6} P_{\mathrm{J}}$ transition between $\mathrm{Gd}^{3+}\left(4 f^{7}\right)$ levels as responsible for the red emission band detected in GaN:Gd.

Although theoretical studies would be required for a reliable identification of the bands, based on these findings we deduced that the bands at 1.77 and $2.02 \mathrm{eV}$ are related to $\mathrm{Gd}$ ions and oxygen vacancy defects located at $\mathrm{Co}^{3+}$ sites, respectively. In order to study the relaxation of the electronic structure across the phase transformation, we assumed the Gd-related feature as a reference due to the difference from that which occurs at the Co-O octahedral/pyramidal sites; thus the local surroundings, as seen by Gd ions, should not vary significantly between the two samples. We calculated the intensity ratio of the Co- to Gd-related emission bands as a function of temperature [inset to Fig. 1(c)]. At room temperature $\delta=0.63$ has a much higher ratio compared to $\delta=0.54$. It is worth recalling that atomic fluctuations give rise to localized levels and/or inhomogeneous charge distribution that can trap electron and holes, increasing the carrier lifetimes [27,28]. PL intensity is sensitive to local disorder degree in the structure, and the observed ratio indicates a more disordered environment seen by $\mathrm{Co}$ in $\mathrm{CoO}_{5} / \mathrm{CoO}_{6}$ coordination for $\delta=0.63$. Upon cooling this ratio decreases to $T=150 \mathrm{~K}$ and correlates with the evolution of the $\eta$ order parameter, suggesting an interplay of structural disorder and a decrease of the orthorhombic strain upon cooling.

Diffuse scattering could potentially provide a direct proof of such disordered character. The lack of single crystals of $\mathrm{GdBaCo}_{2} \mathrm{O}_{5+\delta}$ systems, especially for high $\delta$ values, has resulted in the choice to perform PDF analysis of diffraction powder data. This analysis involves the Fourier transform of the total scattering obtained from the powdered sample containing both the Bragg and diffuse scattering. Nowadays, thanks to the availability of powerful software and the simplicity of data collection, this method is widely adopted to investigate the real structure of disordered oxides. Here we analyzed the structure of $\delta=0.54, \delta=0.63$ samples obtained at $T=300 \mathrm{~K}$ by fitting the observed PDF from $r_{\min }=1.6 \AA$ to higher $r$ by shifting the refinement centroid $\left(r_{\text {mean }}\right)$ [Fig. 3(a)]. The agreement factors $R$ reported in Fig. 3(a) confirm the good quality of the fit at any $r_{\text {mean }}$. We calculate the $r$ dependence of $\eta$ from the lattice parameters obtained at different $r$ ranges. These data are compared in Fig. 3(b) with long-range $\eta$ values obtained by Rietveld (dotted lines). Note that for $\delta=0.54, \eta$ perfectly matches with the distortion of the average structure reflecting the homogeneity of the phase. 

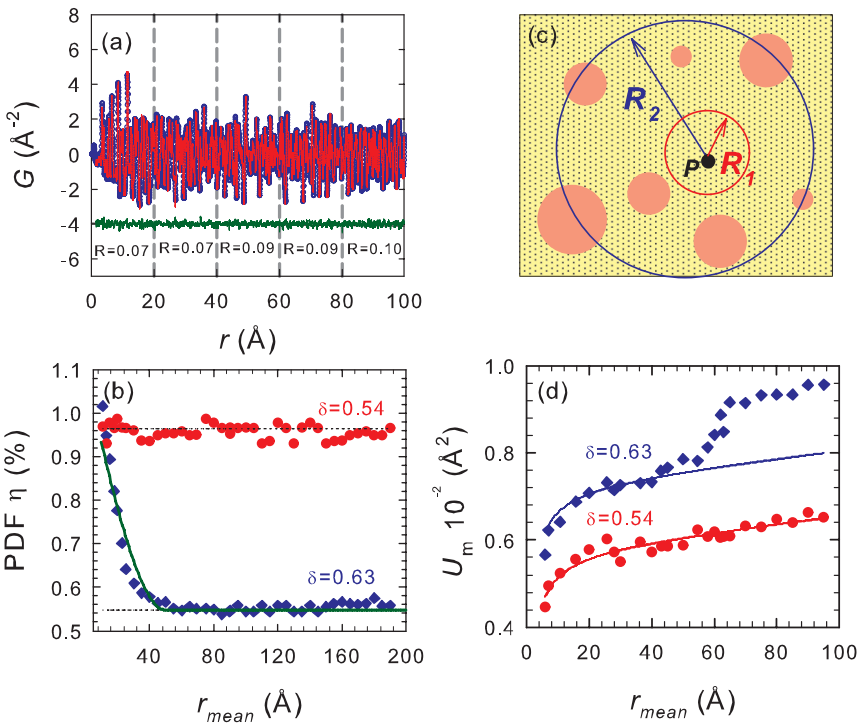

FIG. 3. (Color online) (a) Example of box-car refinement. Vertical dashed lines indicate the boundaries between selected $r$ ranges. (b) PDF refined orthorhombic strain $\eta(\%)$ as a function of centroid mean values of selected $r$ range $\left(r_{\text {mean }}\right)$. Dashed lines are the values of $\eta$ obtained by the Rietveld method and the solid line is the fit to the envelope function. (c) Pictorial view of the proposed $\delta=0.63$ structure containing $\mathrm{Co}_{\mathrm{Co}}^{\bullet}-$ rich regions (full circles) embedded in a LS/IS $\mathrm{Co}^{3+}$-rich matrix (dotted area). (d) $r$ dependence of $U_{\mathrm{m}}$ parameter as defined in the text. In panels (b) and (d), circles and diamonds are data related to $\delta=0.54$ and $\delta=0.63$ samples, respectively.

For $\delta=0.63$ the strong $r$ dependence of the $\eta$ parameter is evident. Locally, $\eta$ is similar to the value found for the $\delta=0.54$ sample, and it falls off rapidly as the fit range approaches the average Rietveld value.

To figure out the $r$ dependence of $\eta$ in $\delta=0.63$, we looked at the interplay of two different possible atomic defects, namely, interstitial oxygen and (low-spin) $\mathrm{LS} \mathrm{Co}^{4+}$ species, generated by increasing $\delta>0.5$. More precisely with increasing $\delta$ the occupancy of the empty $1 c(0,0,1 / 2)$ oxygen site increases and the charge neutrality is satisfied through the formation of defects $\mathrm{O}_{i}^{\prime \prime}, \mathrm{Co}_{\mathrm{Co}}^{\bullet}$ according to the equation $1 / 2 \mathrm{O}_{2}+2 \mathrm{Co}_{\mathrm{Co}}^{x} \rightarrow \mathrm{O}_{i}^{\prime \prime}+2 \mathrm{Co}_{\mathrm{Co}}^{\bullet}$ where, in Kruger-Vink notation [29], $\mathrm{Co}_{\mathrm{Co}}^{x}$ are (intermediate/low-spin) $\mathrm{IS} / \mathrm{LSCo}^{3+}$ located at IS/ $/ \mathrm{LCo}^{3+}$ sites, $\mathrm{O}_{i}^{\prime \prime}$ stands for interstitial oxygen, and $\mathrm{Co}_{\mathrm{Co}}^{\bullet}$ are $\mathrm{LSCo}^{4+}$ located at $\mathrm{IS} / \mathrm{LSCo}^{3+}$ sites. The mutual attraction of these oppositely charged defects drives a formation of clusters between $\mathrm{O}_{i}^{\prime \prime}$ on $\mathrm{Co}_{\mathrm{Co}}^{\bullet}$ sites as neutral defects of type $\left\langle\mathrm{Co}_{\mathrm{Co}}^{\bullet}-\mathrm{O}_{i}^{\prime \prime}-\mathrm{Co}_{\mathrm{Co}}^{\bullet}\right\rangle$. This implies $\mathrm{Co}_{\mathrm{Co}_{0}}^{\bullet}$-rich regions characterized by a decreased $\eta$ parameter, in agreement with structural studies performed on samples with high $\delta$ values [12,14]. In Fig. 3(c) we report a pictorial view of the proposed inhomogeneous pattern where the $\mathrm{Co}_{\mathrm{Co}_{0}}^{\bullet}$-rich regions (red circles) with small $\eta$ exist in the IS $/ \mathrm{LSCo}^{3+}$ rich matrix (dotted area). By considering a generic point $P$ in the real space, one can look at the distribution of the $\eta$ parameter in the sample regions delimited by circles of radii $R_{1}$ and $R_{2}$. We can consider two cases: (1) for $R \leqslant R_{1}, \eta$ coincides with average values of the IS/ $\mathrm{LSCo}^{3+}$ matrix similar to the $\delta=0.54$ sample; (2) for $R \geqslant R_{2}$, different $\eta$ values of the
IS/ $\mathrm{LSCo}^{3+}$ matrix are mixed with $\eta$ belonging to $\mathrm{Co}_{\mathrm{Co}^{\circ}}$-rich regions decreasing the average $\eta$. We can explain the falloff in the amplitude of $\eta$ with increasing $r_{\text {mean }}$ in $\delta=0.63$ by the progressive mixing of $\eta$ parameters of the IS $/ \mathrm{LSCo}^{3+}$ matrix with $\mathrm{Co}_{\mathrm{Co}}^{\bullet}$-rich regions. To estimate the spatial limit below which the IS/ $\mathrm{LSCo}^{3+}$ matrix is separated from the $\mathrm{Co}_{\mathrm{Co}_{0}}^{\bullet}$-rich regions, we fit the $r$ dependence of $\eta$ by using the following relation [solid line in Fig. 3(b)]:

$$
\eta=\eta_{0}+\eta(r, \xi)
$$

where $\eta_{0}$ is the average value of the orthorhombic strain obtained by Rietveld and the second term is a so-called "spherical envelope function" of form [30]

$$
\eta(r, \xi)=A\left[1-\frac{3}{2} \frac{r}{\xi}+\frac{1}{2}\left(\frac{r}{\xi}\right)^{3}\right] \Theta(\xi-r),
$$

where $\xi$ is the diameter defining the spatial limit of the domain, $A$ is a constant, and $\Theta(\xi-r)$ is a step function which is 1 for $r<\xi$ and 0 otherwise. We found $\xi \sim 5 \mathrm{~nm}$, providing evidence of a structural phase separation occurring at the nanoscale. This result is confirmed by the $r$ dependence of the mean atomic displacement parameter $\left(U_{m}\right)$, which is directly related to the mean width of PDF peaks reflecting the static and dynamic disorder of all atom pairs involved [31]. For the $\delta=0.54$ sample, $U_{m}$ follows the low- $r$ peak sharpening due to correlated atom motion [32] and the high- $r$ peak broadening due to the $Q$ dependence of the instrument resolution [32] [Fig. 3(d)]. A clear discontinuity of such behavior is observed at $\delta=0.63$, and the sudden increase of $U_{\mathrm{m}}$ is associated with the enhancement of the width of bond-length distribution due to the mixing of the IS/ $\mathrm{LSCo}^{3+}$ matrix and $\mathrm{Co}_{\mathrm{Co}_{0}}^{\bullet}$-rich regions above $r \sim 5 \mathrm{~nm}$.

The unusual decrease of the orthorhombic strain upon cooling arises from a nanoscale phase-separated structure, which is likely to be caused by a competition of different ordering mechanisms involving charge, orbital, spin, and lattice degrees of freedom. A possible driving force for the structural transition in $\delta=0.63$ could be the magnetic ordering. To validate this hypothesis, we have studied the evolution of the ESR signal as a function of temperature. ESR allows direct access to the spin-environment interactions of Co ions $[4,6]$ and it can be considered a local magnetic probe. ESR spectra for our samples are reported in Figs. 4(a), 4(b) and Figs. 5(a), 5(b). In Figs. 6(a), 6(b) we compared the temperature dependence of ESR linewidth $\left(\Delta H_{\mathrm{pp}}\right)$ with $\chi_{\mathrm{m}}$ data. Details about the ESR signal origin and the procedure to extract $\Delta H_{\mathrm{pp}}$ can be found in our previous studies on $\mathrm{GdBaCo}_{2} \mathrm{O}_{5+\delta}$ systems $[11,12]$.

Our $\chi_{\mathrm{m}}$ curves agree with data reported for similar $\delta$ values [12]. The FM transition takes place at $T_{\mathrm{C}}=279$ and $275 \mathrm{~K}$, whereas the FM phase suddenly disappears at $T_{\mathrm{N} 1}=256$ and $253 \mathrm{~K}$ for the $\delta=0.54$ and 0.63 samples, respectively, where the AFM1 phase arises. These transition temperatures are close to the data reported for the $\delta=0.5$ reference sample [12].

$\Delta H_{\mathrm{pp}}$ markedly changes across the transitions at $T_{\mathrm{C}}$ and $T_{\mathrm{N} 1}$. In particular, for $\delta=0.54$ at room temperature the observed absorption is consistent with the broadening of PM $\mathrm{Gd}^{3+}$ resonance due to exchange interactions between $\mathrm{Gd}$ localized $4 f$ orbital and Co spins [4,6]. On approaching $T_{\mathrm{C}}$, a 


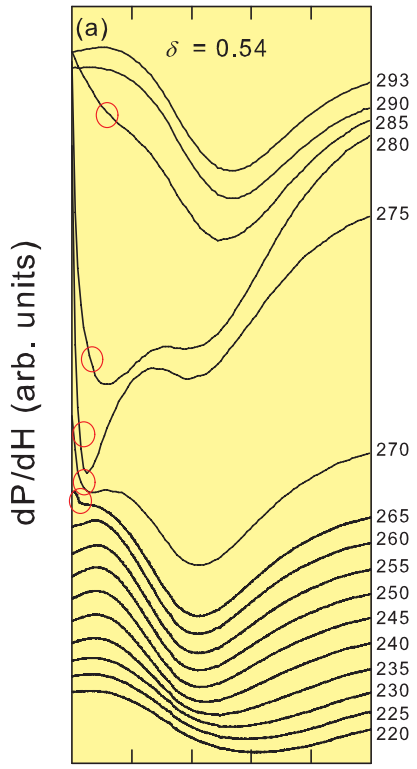

$\begin{array}{llllll}0.0 & 0.2 & 0.4 & 0.6 & 0.8 & 1.0\end{array}$

$\mathrm{H}(\mathrm{T})$

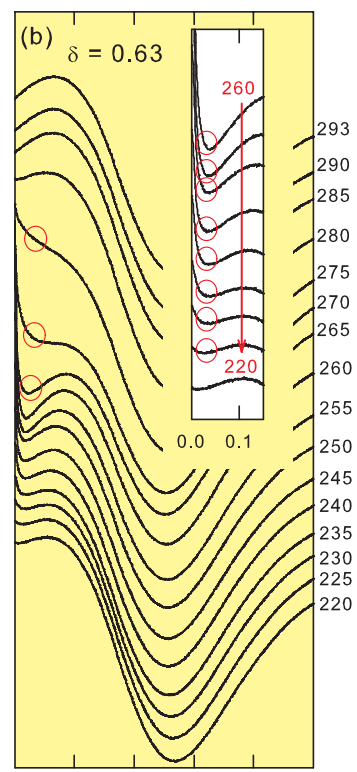

$\begin{array}{llllll}0.0 & 0.2 & 0.4 & 0.6 & 0.8 & 1.0\end{array}$

$\mathrm{H}(\mathrm{T})$

FIG. 4. (Color online) Temperature evolution of ESR spectra for samples (a) $\delta=0.54$ and (b) $\delta=0.63$. Inset to (b) shows a magnified view of the low-field part of the spectra. Circular markers highlight the emergence of FMR below room temperature.

second resonance emerges at lower fields, altering the spectra shape [Fig. 4(a)]. This signal is due to the ferromagnetic resonance (FMR) generated by the ordering of unpaired spins on Co ions. Below $T_{\mathrm{C}}$, the Co FMR shifts to lower fields,

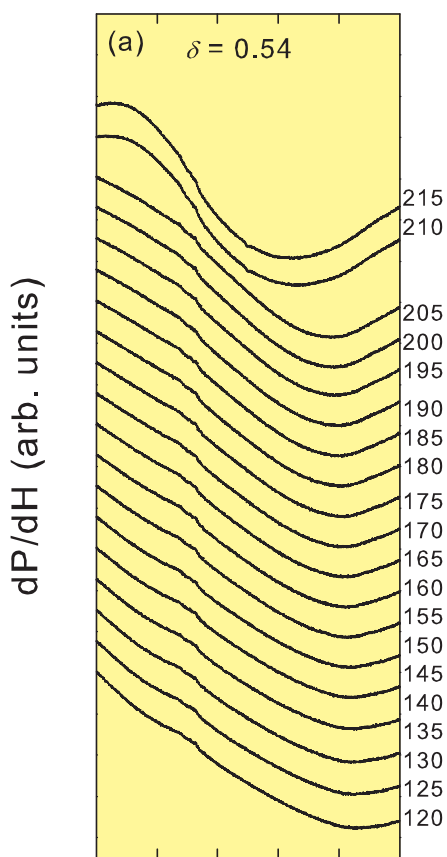

$\begin{array}{llllll}0.0 & 0.2 & 0.4 & 0.6 & 0.8 & 1.0\end{array}$

$\mathrm{H}(\mathrm{T})$

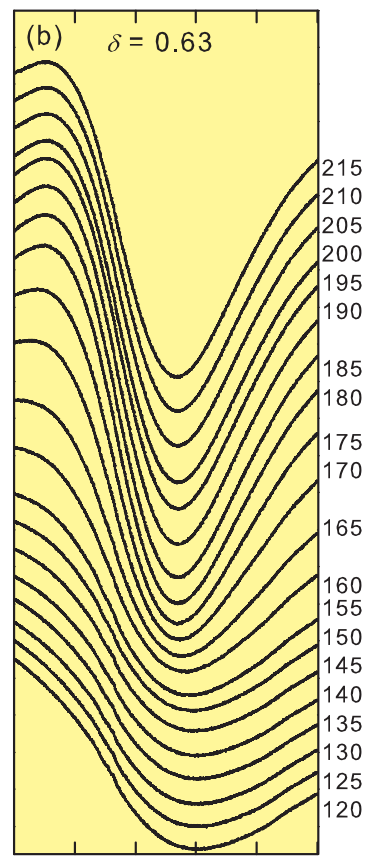

$\begin{array}{llllll}0.0 & 0.2 & 0.4 & 0.6 & 0.8 & 1.0\end{array}$

$\mathrm{H}(\mathrm{T})$

FIG. 5. (Color online) Temperature evolution of ESR spectra at low temperature for samples (a) $\delta=0.54$ and (b) $\delta=0.63$.

indicating an increase of an internal field corresponding to the temperature dependence of the FM magnetization in $\chi_{\mathrm{m}}$ [Fig. 6(b)]. FMR disappears at around $T_{\mathrm{N} 1}$, and a single broad resonance line dominates the ESR signal. Upon cooling, the signal displays a fast broadening, i.e., $\Delta H_{\mathrm{pp}}$ increases, down to $T \sim 210 \mathrm{~K}$. Upon further cooling, the ESR signal totally disappears [Fig. 5(a)], indicating the emergence of AFM correlations acting upon the $\mathrm{Gd}^{3+}$ resonance. This critical temperature matches with $T_{\mathrm{N} 2}$ of the AFM1-AFM2 transition inferred from muon spin relaxation measurements in cobaltites with $\delta \sim 0.5$ [13]. Here we provide evidence that the same transition occurs in GBCO at $T_{\mathrm{N} 2} \sim 210 \mathrm{~K}$. This is also confirmed by the fast broadening of the ESR linewidth observed above $T_{\mathrm{N} 2}$ described by the critical equation $[4,33]$ $\Delta H_{\mathrm{pp}}=a\left[\left(T-T_{\mathrm{N} 2}\right) / T_{\mathrm{N} 2}\right]^{-m}$, where $a$ and $m$ are constants. Experimental data were fitted in the two linear regimes, with $m=-0.5$ and -0.1 , observed in the log-log plot of $\Delta H_{\mathrm{pp}}$ [inset to Fig. 6(a)]. The fitted $m$ values are consistent with the temperature dependence of $\Delta H_{\mathrm{pp}}$ expected when AFM spin fluctuations, precursors of transition at $T_{\mathrm{N} 2}$, occur [4].

For the $\delta=0.63$ sample, the emergence of FMR below $T_{\mathrm{C}}$ is concomitant with the evolution of FM magnetization [Fig. 4(b)]. Below $T=260 \mathrm{~K}$, FMR shifts to lower field values but it does not disappear below $T_{\mathrm{N} 1}$ [inset to Fig. 4(b)]. This indicates the persistence of the FMR internal field even

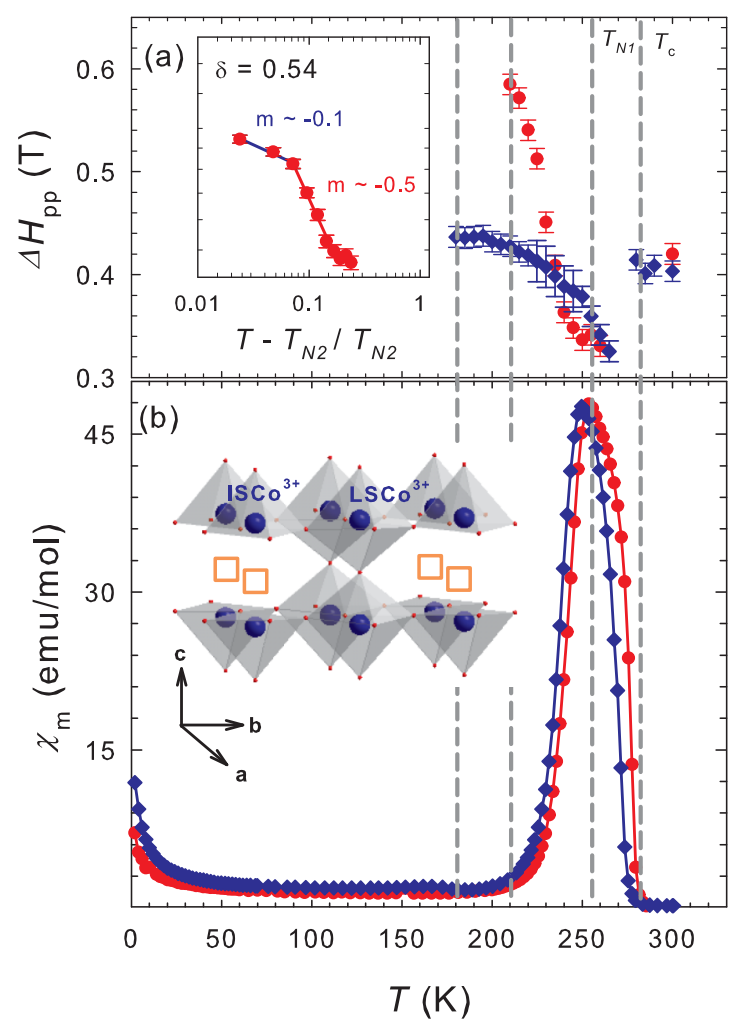

FIG. 6. (Color online) (a) Temperature evolution of $\Delta H_{\mathrm{pp}}$ for $\delta=0.54$ (circles), $\delta=0.63$ (diamonds). Inset to (a) shows the log-log plot of $\Delta H_{\mathrm{pp}}$ for $\delta=0.54$. (b) Magnetic susceptibility of $\delta=0.54$ (circles), $\delta=0.63$ (diamonds) samples, as a function of temperature collected at applied field $H=20 \mathrm{Oe}$. Inset to (b) shows the crystal structure of GBCO with the oxygen site $(0,0,1 / 2)$ (squares) fully unoccupied. The alternation of $\mathrm{ISCo}^{3+}$ and $\mathrm{LSCo}^{3+}$ located at $\mathrm{CoO}_{5}$ and $\mathrm{CoO}_{6}$ units is shown. $\mathrm{Ba}$ and $\mathrm{Gd}$ are omitted for clarity. 
when, in the same $253 \mathrm{~K} \leqslant T \leqslant 220 \mathrm{~K}$ range, the dropping of the FM component in $\chi_{\mathrm{m}}$ is consistent with AFM ordering. Below $T=220 \mathrm{~K}$ the low-field signal vanishes and the signal consists of a single broad $\mathrm{Gd}^{3+}$ resonance line [Fig. 5(b)]. Fast broadening of the ESR spectrum is observed below $T_{\mathrm{N} 1}$, but for $T<240 \mathrm{~K}, \Delta H_{\mathrm{pp}}$ saturates down to $T \sim 180 \mathrm{~K}$. For $T<180 \mathrm{~K}$ the $\mathrm{Gd}^{3+}$ signal starts to be distorted [Fig. 5(b)], indicating the emergence of strong AFM spin correlations. We argue that the AFM1-AFM2 transition takes place in $\delta=0.63$ at $T_{\mathrm{N} 2}=180 \mathrm{~K}$.

The persistence of FMR in the AFM1 regime provides an indication of magnetic phase coexistence in the $\delta=0.63$ sample. To figure out the nature of this inhomogeneity, we consider the interactions of $\mathrm{ISCo}^{3+}$ located at $\mathrm{CoO}_{5}$, forming two-leg ladders along the $a$ axis and separated by $a c \mathrm{CoO}_{2}$ layers composed of nonmagnetic $\mathrm{LSCo}^{3+}$ located at $\mathrm{CoO}_{6}$ $[11,12]$, as sketched in inset of Fig. 6(b). As proposed for the $\delta=0.54$ sample [6], the FM order below $T_{\mathrm{C}}$ can be explained by the intraladder interactions of orbitally ordered $\mathrm{IS} \mathrm{Co}^{3+}$ ions, while the interladder interactions are dominated by AFM $\mathrm{ISCo}^{3+}-\mathrm{ISCo}^{3+}$ superexchange (SE) interactions mediated by spinless $\mathrm{CoO}_{6}$ layers [inset to Fig. 6(b)] [11,12]. The increased number of $\mathrm{Co}_{\mathrm{Co}}^{\circ}$ upon doping $\mathrm{O}_{i}^{\prime \prime}$ into $(0,0,1 / 2)$ sites enhances the double-exchange (DE) FM interactions along the $\mathrm{LSCo}^{4+}$ O-ISCo ${ }^{3+}$ DE paths. FMR below $T_{\mathrm{C}}$ is consistent with the intraladder FM ordering, but the persistence of FMR about $30 \mathrm{~K}$ below $T_{\mathrm{N} 1}$ can be ascribed to DE paths competing with SE interladder channels in $\delta=0.63$. By considering the collective interactions of strong DE paths, we can visualize the magnetic inhomogeneity pattern as a distribution of small FM clusters embedded within the AFM1 phase.

Having identified the nature of the magnetic inhomogeneity in $\delta=0.63$, we will now discuss its interplay with the unusual decrease of the orthorhombic strain upon cooling. In principle, the excess of oxygen $(\delta>0.5)$ in the $\mathrm{LnO}_{\delta}$ plane involves a redistribution of oxygen ions, which breaks the Pmmm symmetry into a vacancy disordered phase with $P 4 / \mathrm{mmm}$ space group and $2 a \times 2 a \times 2 a$ cell metric, as observed, e.g., in $\mathrm{PrBaCo}_{2} \mathrm{O}_{5+\delta}$ for $\delta=0.74$ [14]. The structure of our $\delta=0.63$ sample is still consistent with the Pmmm structure at room temperature and, since we did not find any further superlattice reflections, we ruled out the occurrence of a long-range order-disorder mechanism in the $\mathrm{GdO}_{0.63}$ plane. On the other hand, we found that our PDF analysis is consistent with the clustering, i.e., short-range ordering, of the doped $\mathrm{O}_{i}^{\prime \prime}$ in $\delta=0.63$ which are no longer randomly distributed, inducing a $\mathrm{Co}_{\mathrm{Co}}^{-}$-rich region in the IS/LS $\mathrm{Co}^{3+}$ matrix already at room temperature. The interactions between the $\mathrm{Co}_{\mathrm{Co}}^{\bullet}$-rich regions and the host matrix generate strong $\mathrm{DE}$ $\mathrm{LSCo}^{4+}-\mathrm{O}-\mathrm{ISCo}^{3+}$ paths, which accounts for the formation of FM clusters embedded in a globally AFM1 phase dominated by $\mathrm{ISCo}^{3+}-\mathrm{O}-\mathrm{ISCo}^{3+} \mathrm{SE}$ interactions. The difference of structural parameters between the two phases gives rise to change of $\eta$ from one sample region to another, reducing the average $\eta$. Upon cooling, the thermal contraction of both the $a$ axis and $b$ axis in the IS/LSCo ${ }^{3+}$ matrix and in $\mathrm{Co}_{\mathrm{Co}}^{\bullet}$-rich regions induces a decrease of $\eta$ magnitude across the FM-AFM1 transition. At around $T_{\mathrm{N} 2}=180 \mathrm{~K}$, the emergence of the AFM2 phase competes with FM $\mathrm{LSCo}^{4+}-\mathrm{O}-\mathrm{ISCo}^{3+}$ paths and causes the suppression of FMR already about

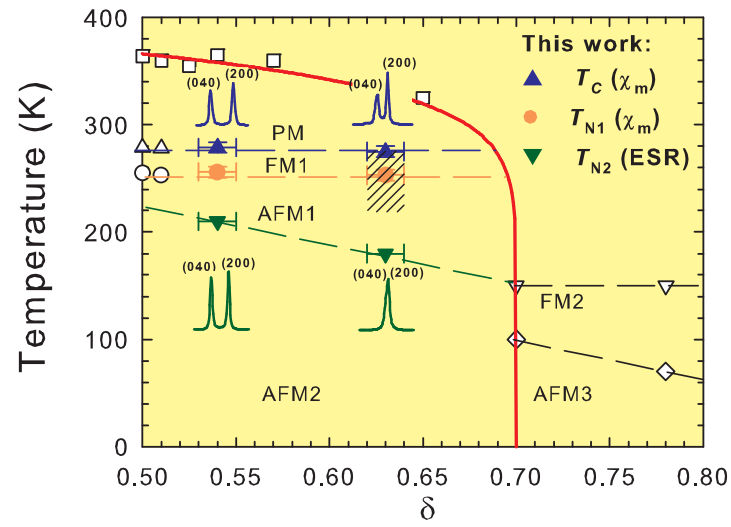

FIG. 7. (Color online) Phase diagram proposed for $\mathrm{GdBaCo}_{2} \mathrm{O}_{5+\delta}$ in the $0.5 \leqslant \delta \leqslant 0.77$ region. Empty symbols are data taken from Refs. [6,11,12]. Insets at $\delta=0.54, \delta=0.63$ show portions of powder diffraction patterns at $T=300 \mathrm{~K}$ and $T=150 \mathrm{~K}$. For $\delta=0.63$ the change of the spectrum due to the dramatic decrease of the orthorhombic strain going from the PM to AFM2 phases on cooling is clearly shown. Solid line indicates the boundary of MIT.

$40 \mathrm{~K}$ above the magnetic transition. As observed for $\delta=0.54$ below $T \sim 100 \mathrm{~K}$ [Fig. 1(c)], for $\delta=0.63$ quantum zero-point motion induces a flattening of $\eta$ that reaches a broad minimum between 180 and $150 \mathrm{~K}$. As a matter of fact, below these temperatures the thermal vibrations are no longer able to mediate the fluctuations in the primary order parameter, i.e., $\eta$, damping the competition among the differently strained sample regions. The distortion of the main phase is then restored, resulting in a smooth increase of $\eta$. This further low- $T$ reentrant transition is even more evident by looking at the diffraction patterns shown in Fig. 1(a) below $T=150 \mathrm{~K}$.

\section{CONCLUSION}

To summarize the results of our study, in Fig. 7 we report the obtained critical temperatures within the $T-\delta$ phase diagram of GBCO for $0.5 \leqslant \delta \leqslant 0.77[6,11,12]$. We found an AFM2 phase able to totally quench the strong $\mathrm{Gd}^{3+} \mathrm{ESR}$ resonance below $T_{\mathrm{N} 2}$. Additionally, for $\delta=0.63$ we uncovered an inhomogeneous magnetic phase where FM clusters are embedded in an AFM1 matrix phase in a narrow temperature range below $T_{\mathrm{N} 1}$. This result is fully supported by local structural analysis, which suggests a nanoscale phase separation between $\mathrm{Co}_{\mathrm{Co}}^{\circ}$-rich regions and the $\mathrm{IS} / \mathrm{LSCo}^{3+}$ matrix, rather than a long-range phase separation as previously reported [12]. Finally, as a consequence of such nanoscale phase separation, we observed a dramatic decrease of the orthorhombic strain upon cooling in the average structure of the $\delta=0.63$ sample across the FM-AFM1-AFM2 transition series. This work shows unambiguously that doping GBCO above the optimal doping value $(\delta \sim 0.5)$ induces structural and magnetic nanoscale inhomogeneities which dramatically destabilize the orthorhombic structure below room temperature.

\section{ACKNOWLEDGMENTS}

The authors gratefully acknowledge the European Synchrotron Radiation Facility for provision of beam time and Dr. Adrian Hill for assistance in using the ID31 beamline. 
[1] E. Dagotto, Science 309, 257 (2005).

[2] E. Dagotto, in Nanoscale Phase Separation and Colossal Magnetoresistance (Springer, Berlin, 2003).

[3] M. Allieta, M. Scavini, L. J. Spalek, V. Scagnoli, H. C. Walker, C. Panagopoulos, S. S. Saxena, T. Katsufuji, and C. Mazzoli, Phys. Rev. B 85, 184107 (2012).

[4] M. Allieta, M. Scavini, L. Lo Presti, M. Coduri, L. Loconte, S. Cappelli, C. Oliva, P. Ghigna, P. Pattison, and V. Scagnoli, Phys. Rev. B 88, 214104 (2013).

[5] L. Lo Presti, M. Allieta, M. Scavini, P. Ghigna, L. Loconte, V. Scagnoli, and M. Brunelli, Phys. Rev. B 84, 104107 (2011).

[6] M. Allieta, C. Oliva, M. Scavini, S. Cappelli, E. Pomjakushina, and V. Scagnoli, Phys. Rev. B 84, 235144 (2011).

[7] T. Vogt, P. M. Woodward, P. Karen, B. A. Hunter, P. Henning, and A. R. Moodenbaugh, Phys. Rev. Lett. 84, 2969 (2000).

[8] A. Maignan, V. Caignaert, B. Raveau, D. Khomskii, and G. Sawatzky, Phys. Rev. Lett. 93, 026401 (2004).

[9] D. Chernyshov, G. Rozenberg, E. Greenberg, E. Pomyakushina, and V. Dmitriev, Phys. Rev. Lett. 103, 125501 (2009).

[10] A. A. Taskin and Y. Ando, Phys. Rev. Lett. 95, 176603 (2005).

[11] A. A. Taskin, A. N. Lavrov, and Y. Ando, Phys. Rev. Lett. 90, 227201 (2003).

[12] A. A. Taskin, A. N. Lavrov, and Y. Ando, Phys. Rev. B 71, 134414 (2005).

[13] H. Luetkens, M. Stingaciu, Yu. G. Pashkevich, K. Conder, E. Pomjakushina, A. A. Gusev, K. V. Lamonova, P. Lemmens, and H.-H. Klauss, Phys. Rev. Lett. 101, 017601 (2008).

[14] C. Frontera, A. Caneiro, A. E. Carrillo, J. Oró-Solé, and J. L. Garcı́́-Munõz, Chem. Mater. 17, 5439 (2005).

[15] C. Frontera, J. L. Garcia-Munoz, C. Ritter, and A. Canerio, J. Phys.: Condens. Matter 20, 104228 (2008).

[16] D. P. Kozlenko, Z. Jirák, N. O. Golosova, and B. N. Savenko, Eur. Phys. J. B 70, 327 (2009).

[17] A. C. Larson and R. B. Von Dreele, General Structure Analysis System (GSAS), Los Alamos National Laboratory Report LAUR 86-748 (2004).

[18] X. Qiu, J. W. Thompson, and S. J. L. Billinge, J. Appl. Cryst. 37, 678 (2004).
[19] C. L. Farrow, P. Juhás, J. W. Liu, D. Bryndin, E. S. Božin, J. Bloch, Th. Proffen, and S. J. L. Billinge, J. Phys.: Condens. Matter 19, 335219 (2007).

[20] Yu. G. Pashkevich, V. P. Gnezdilov, P. Lemmens, K. Y. Choi, K. V. Lamonova, A. A. Gusev, G. Güntherodt, S. N. Barilo, S. V. Shiryaev, and G. L. Bychkov, Spectroscopy of Emerging Materials 165, 195 (2004).

[21] T. P. J. Han, M. Villegas, M. Peiteado, A. C. Caballero, F. Rodríguez, and F. Jaque, Chem. Phys. Lett. 488, 173 (2010).

[22] M. Anpo and Y. Kubokawa, J. Phys. Chem. 88, 5556 (1984).

[23] G. Faggio, V. Modafferi, G. Panzera, D. Alfieri, and S. Santangelo, J. Raman Spectrosc. 43, 761 (2012).

[24] A. Naldoni, M. Allieta, S. Santangelo, M. Marelli, F. Fabbri, S. Cappelli, C. L. Bianchi, R. Psaro, and V. Dal Santo, J. Am. Chem. Soc. 134, 7600 (2012).

[25] V. N. Kuznetsov and N. Serpone, J. Phys. Chem. C 113, 15110 (2009).

[26] B. Witek, A. Wysmołek, M. Kamińska, A. Twardowski, M. Potemski, M. Boćkowski, and I. Grzegory, Acta Phys. Pol., A 114, 1425 (2008).

[27] F. V. Motta, A. T. de Figueiredo, V. M. Longo, V. R. Mastelaro, A. Z. Freitas, L. Gomes, N. D. Vieira, Jr., E. Longo, and J. A. Varela, J. Lumin. 129, 686 (2009).

[28] S. de Lazaro, J. Milanez, A. T. de Figueiredo, V. M. Longo, V. R. Mastelaro, F. S. De Vicente, A. C. Hernandes, J. A. Varela, and E. Longo, Appl. Phys. Lett. 90, 111904 (2007).

[29] F. A. Kröger and H. J. Vink, Solid State Phys. 3, 307 (1956).

[30] R. C. Howell, T. Proffen, and S. D. Conradson, Phys. Rev. B 73, 094107 (2006).

[31] T. Egami and S. J. L. Billinge, Underneath the Bragg Peaks: Structural Analysis of Complex Materials, Pergamon Materials Series Vol. 7 (Pergamon, Oxford, 2003).

[32] X. Qiu, E. S. Božin, P. Juhás, Th. Proffen, and S. J. L. Billinge, J. Appl. Cryst. 37, 110 (2004).

[33] C. Filip, L. Giurgiu, X. Filip, C. Kessler, and M. Mehring, Czech. J. Phys. 46, 1143 (1996). 